\title{
Euroroundup
}

\section{TraVel-associated Legionnaires' Disease In EUROPE: 2005}

\author{
KD Ricketts, B McNaught, CA Joseph, on behalf of the European Working Group for Legionella Infections
}

Respiratory Diseases Department, Health Protection Agency Centre for Infections, London, United Kingdom

In 2005, 755 cases of travel-associated legionnaires' disease with onset in 2005 were reported to the EWGLINET surveillance scheme by 20 countries. A total of $85.8 \%$ of cases were diagnosed by the urinary antigen test, and 37 cultures were obtained. Twenty-nine deaths were reported, giving a case fatality rate of $3.8 \%$ (down from $5.6 \%$ in 2004). Ninety three new clusters were identified, $36.6 \%$ of which would not have been detected without the EWGLINET scheme. One hundred and twenty two accommodation sites were investigated and the names of nine sites were published on the EWGLI website. Thirty-two sites were associated with additional cases after a report was received to say that investigations and control measures had been satisfactorily carried out. This level of re-offending is greater than in previous years and care should be taken to ensure the guidelines are being properly applied, especially in Turkey.

\section{Introduction}

Following the emergence of legionnaires' disease in 1976, the nations of Europe formed the European Working Group for Legionella Infections (EWGLI), a network of public health professionals from countries across the continent aiming to facilitate collaborations. The following year, EWGLI established a surveillance scheme for travel-associated legionnaires' disease designed to track all cases of the disease in European travellers. This scheme became known as EWGLINET and, in 2002, adopted the European Guidelines for Control and Prevention of Travel Associated Legionnaires' Disease (more commonly referred to as 'the European guidelines') [1].

When EWGLINET suspects that a cluster of cases is associated with an accommodation site, the network initiates and monitors immediate control measures and investigations at the site, ensuring that international standards are adhered to. Further information on the activities of EWGLI can be found on its website (http://www. ewgli.org).

This paper provides results and commentary on cases of travelassociated legionnaires' disease reported to EWGLINET with onset in 2005.

\section{Methods}

During 2005 the Former Yugoslav Republic of Macedonia joined EWGLINET, while Tunisia left the scheme when their collaborator moved on and a replacement could not be identified. As a result, the number of collaborating countries remained stable at 35 [Figure 1]. (In previous publications this figure has counted the separate reporting areas of the United Kingdom - England \& Wales, Northern Ireland, and Scotland - as three separate countries. The total given here counts the UK as one country.) These 35 countries inform EWGLINET of any travel-associated case of legionnaires' disease detected by their national surveillance systems, if it fulfils EWGLI's

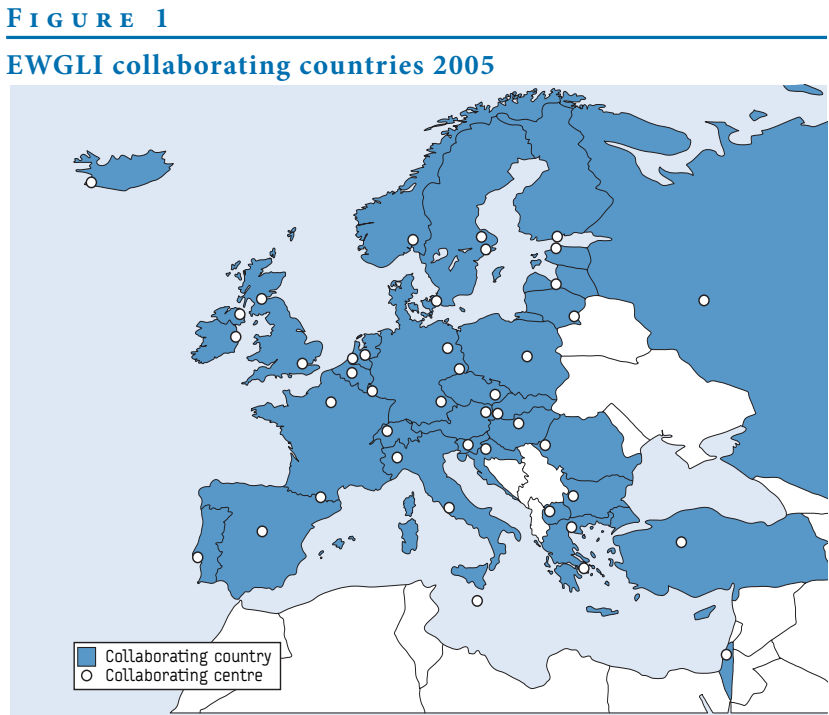

case definitions. This includes cases in patients who travelled within their own countries as well as those who travelled abroad.

Standard case definitions have been agreed by the collaborating countries in EWGLI and are used for the purposes of international surveillance [1]. When a case of travel-associated legionnaires' disease is detected by a national surveillance scheme, the case is reported to EWGLI as described in previous publications [2].

The European guidelines require minimal site investigations when the case is not part of a cluster, because the individual may have had multiple exposures during his or her incubation period, and therefore there is little evidence that the accommodation site is the source of the infection. However, collaborators are expected to issue a 14 point checklist to the site(s) to ensure that the risk of legionella infection is minimised.

For cases associated with clusters, more extensive investigations are required. Within two weeks, a 'Form A' must be completed and sent to the coordinating centre, stating that a risk assessment has been carried out and that control measures are in progress. Within a further four weeks (six weeks in total) a 'Form B' must be received, stating that control measures and sampling have been carried out, giving the results of the sampling, and saying whether the accommodation site remains open or has been closed. If these forms are not returned to the coordinating centre within the time allowed, EWGLINET will publish the details of the site on its public website (http://www.ewgli.org). Once the collaborator of the country 
of infection returns the relevant form(s), confirming that measures to control the risk of legionella infection at the site have been taken, this notice is removed from the website [1].

\section{Results}

Cases and outcomes

Seven hundred and fifty-two cases of travel-associated legionnaires' disease with onset during 2005 were reported by 18 of the 35 collaborating countries. In addition, Australia and the United States, reported two and one cases respectively even though they are not official collaborating countries. This easily surpasses the previous highest annual total of cases from 2002 (677 cases). Echoing the pattern of recent years, the countries that reported most cases in 2005 were the UK (202 cases), France (157), the Netherlands (134) and Italy (96) [Table 1].

\begin{tabular}{lllll} 
T A B L & $\mathbf{1}$ \\
\hline
\end{tabular}

Countries reporting more than 10 cases of travel-associated legionnaires' disease to EWGLINET in 2005

\begin{tabular}{|c|c|}
\hline Country of report & Number of cases \\
\hline United Kingdom & 202 \\
\hline France & 157 \\
\hline The Netherlands & 134 \\
\hline Italy & 96 \\
\hline Denmark & 40 \\
\hline Spain & 30 \\
\hline Sweden & 23 \\
\hline Austria & 18 \\
\hline Belgium & 13 \\
\hline Norway & 13 \\
\hline
\end{tabular}

NB: In addition, 10 other countries reported fewer than 10 cases, and are not listed here

The distinctive age and gender profile seen in previous years was repeated in 2005, with male cases outnumbering female cases by a significant margin (a ratio of 2.5:1, down from 2.9:1 in 2004 but still consistent with longer term trends), and a skewing of cases towards older age groups (with peaks in the 60-69 years group for both sexes). The median age for male cases was 59 years (age range $21-93$ years) and for female cases was 60 years (age range 13-86 years).

As is to be expected for a travel-associated scheme, the established pattern of a seasonal peak in summer was again seen in 2005, but this time with a peak in September, slightly later than the August peak that occurred in 2004.

There was a significant drop in the case fatality rate in 2005 , which fell to $3.8 \%$ (from $5.6 \%$ in 2004). The 29 deaths reported to EWGLINET in 2005, together with the 316 cases who reportedly recovered from their illness, are considered to be the 'known' outcomes (45.7\% in 2005 compared with $47.2 \%$ in 2004). This contrasts with the 410 cases whose outcome is 'unknown' (54.3\%). This proportion is smaller than in 2004 (52.8\%) but more significant than in 2003 (44\%) or 2002 (36.1\%). This suggests that the ratio of 'known' to 'unknown' outcomes has stabilised over recent years, despite efforts made each year to improve the reporting of final outcomes for cases.
F I G U R E 2

Countries visited by more than 10 cases of travel-associated legionnaires' disease in 2005 , by type of case

Single cases

Cluster cases

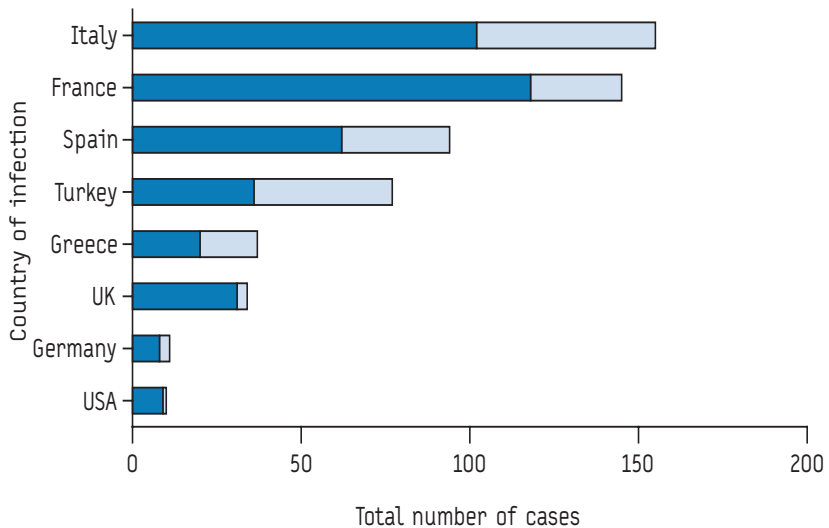

The 29 reported deaths were in individuals aged between 30 and 85 years. Twenty-four were male (83\%, compared with $81 \%$ in 2004 ) and five were female (17\%). Twenty-one of the deaths were associated with single cases (72\%), and the remaining eight were associated with cluster cases (28\%, compared with $32 \%$ in 2004).

\section{Microbiology}

Urinary antigen detection was the main method of diagnosis in the majority of cases in 2005 (85.8\%). This is an increase on the proportion of $84.9 \%$ in 2004 , which was itself an increase on previous years. The use of serology as the main method of diagnosis continued to decrease as in previous years, falling to $7.0 \%$ in 2005 ( $8.7 \%$ in 2004$) ; 2.5 \%$ by fourfold rise (3.7\% in 2004 ) and $4.5 \%$ by single high titre $(5.0 \%$ in 2004$)$. The number of culture proven cases stayed constant at 37 (the same as in 2004), but the increased activity in 2005 meant that the culture diagnoses represented a smaller proportion of the total than in $2004(4.9 \%$ in 2005 down from $5.6 \%$ in 2004). Seventeen cases (2.3\%) were diagnosed primarily by other methods (up from $0.8 \%$ in 2004).

The main category of organism detected in 2005 was 'Legionella pneumophila serogroup 1' (625 cases, $82.8 \%$ compared with $69.3 \%$ in 2004). The remaining cases were reported as ' $L$. pneumophila other serogroup' (15 cases, $2.0 \%$; $2.0 \%$ in 2004 ), 'L. pneumophila serogroup unknown' (88 cases, $11.7 \%$; $23.5 \%$ in 2004), 'Legionella, other species' (1 case, $0.1 \%$; $0.3 \%$ in 2004), and 'Legionella, species unknown' (26 cases, 3.4\%; $4.9 \%$ in 2004).

\section{Travel}

Cases visited 64 different countries during their incubation periods. Seventy-nine cases $(10.5 \%)$ visited countries outside the EWGLINET scheme. Forty-nine cases visited more than one European country, and eight visited more than one country outside Europe.

The proportion of cases associated with travel to the four main countries of infection increased substantially to $62 \%$ in 2005 
(from $53 \%$ in 2004 ): Italy - 155 cases ( $21 \%$ of all cases), France - 145 cases (19\%), Spain - 94 cases (12\%), and Turkey - 77 cases (10\%). A large proportion of the cases visiting sites in France were French nationals (94 cases) travelling internally in their own country, and likewise with Italian nationals visiting sites in Italy (83 cases). Spain did not report any of their nationals who acquired disease after travelling within Spain, and neither were any Turkish cases reported who had travelled within their own country.

Nineteen percent of cases travelling to France were associated with clusters; for cases involving travel to Spain and Italy the figure was $34 \%$ for each, while for Turkey it was 53\% [Figure 2] (up from $44 \%$ in 2004 , although still much lower than the $71 \%$ of cases in Turkey which were associated with clusters in 2002).

\section{Clusters}

Ninety-three new clusters were identified in 2005, compared with 86 in 2004, 89 in 2003 and 94 in 2002 (this does not include clusters which were identified in previous years and were associated with a subsequent case in 2005; these clusters are included in the previous years' figures). Sixty seven clusters were composed of only two cases; the proportion of clusters involving only two or three cases reached $93.5 \%$ [Figure 3], compared with almost $90 \%$ in 2004. The largest cluster in 2005 involved eight cases (up from six cases in 2004). Of the 93 new clusters, 34 consisted of a single case reported by each of two or more countries. These clusters would not have been detected without EWGLINET because national surveillances schemes do not ordinarily detect clusters that involve fewer than two of their citizens.

The clusters in 2005 were located in 20 countries, and one cluster was associated with a cruise ship. Italy was associated with the most clusters (27), followed by Turkey (15), France (14), Greece (8), and Spain (8) [Table 2]. Of the remaining clusters, 14 occurred in countries outside EWGLINET, or in EWGLINET countries not officially signed up to follow the European guidelines. This represents $15 \%$ of clusters, a slight drop on the $16 \%$ seen in 2004. Ten clusters involved two or more accommodation sites.

Clusters were detected during every month of 2005 (by date of onset of the second case in the cluster). Most of the clusters arose

F I G U R E 3

Number of cases of travel-associated legionnaires' disease per cluster, by year, from 2002 to 2005

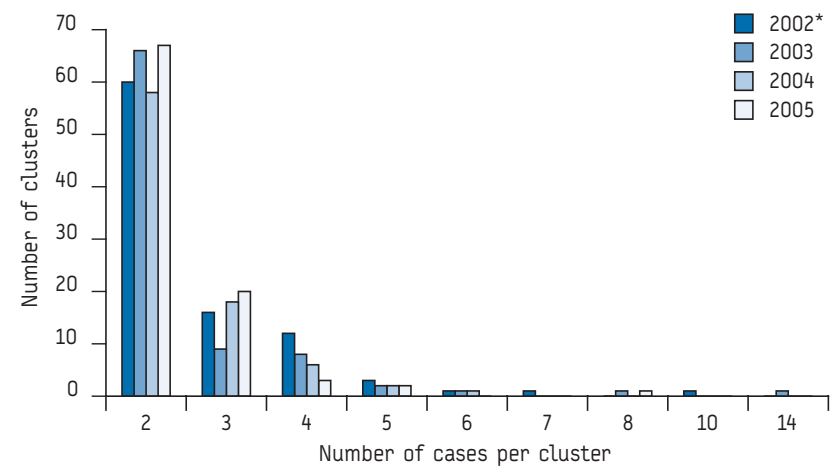

* 2002 figures include clusters both pre and post guidelines [1], adopted $1^{\text {st }}$ July 2002
TA B L E 2

Countries associated with clusters of travel-associated legionnaires' disease in 2005

\begin{tabular}{|c|c|c|}
\hline & Country of infection & Number of clusters \\
\hline \multicolumn{3}{|l|}{ European Union } \\
\hline & Italy & 27 \\
\hline & France & 14 \\
\hline & Greece & 8 \\
\hline & Spain & 8 \\
\hline & Germany & 2 \\
\hline & Cyprus* & 1 \\
\hline & Czech Republic & 1 \\
\hline & $\begin{array}{l}\text { United Kingdom } \\
\text { (England) }\end{array}$ & 1 \\
\hline & United Kingdom (Wales) & 1 \\
\hline \multicolumn{3}{|l|}{ Non-European Union } \\
\hline & Turkey & 15 \\
\hline & Egypt & 3 \\
\hline & Thailand & 2 \\
\hline & Antigua & 1 \\
\hline & Dominican Republic & 1 \\
\hline & India & 1 \\
\hline & Malaysia & 1 \\
\hline & Morocco & 1 \\
\hline & Netherlands Antilles & 1 \\
\hline & Philippines & 1 \\
\hline & Tanzania & 1 \\
\hline & Tunisia & 1 \\
\hline \multicolumn{3}{|l|}{ Other } \\
\hline & Ship & 1 \\
\hline
\end{tabular}

*Areas not under the effective control of the Government of the Republic of Cyprus

during the summer months, with $67 \%$ occurring between May and September (62 clusters).

\section{Investigations and publications}

The 93 new clusters in 2005 involved a total of 104 accommodation sites. Of these, 15 were located in countries not signed up to follow the European guidelines, leaving 89 of these new cluster sites that required EWGLINET investigations. In addition, 32 sites that had been involved in previous clusters which were investigated under the guidelines were associated with extra cases during 2005 ('cluster updates'); the guidelines require that these be investigated again. These sites are known as 're-offending' sites. Also, one site in Hungary that had been involved in a cluster previously was associated with an extra case in 2005; this site had not been investigated earlier under the guidelines, but by 2005 Hungary was following the European guidelines and investigated the cluster update accordingly.

Therefore, in 2005 EWGLINET requested the investigation of a total of 122 sites. A positive sampling result (concentrations of Legionella equal to or greater than $1,000 \mathrm{cfu} / \mathrm{litre}$ [1]) was reported on 70 of the 'Form B' reports returned to the coordinating centre 
(57.4\%), 50 (41.0\%) reported that Legionella was not detected in samples at the required levels, and two 'Form B' reports (1.6\%) did not have samples taken for reasons accepted by the coordinating centre.

Of the re-offending sites, six re-offended twice during 2005, giving 26 distinct sites that were associated with further cases subsequent to a previous cluster (compared with 18 distinct sites in 2004, one of which re-offended twice). Eleven of these sites were situated in Turkey (compared with only four in 2004), five in Italy, three each in France and Spain, two in Greece and one in each of, Malta and Poland. Between them, these sites were subject to 32 reinvestigations and 16 returned positive samples (compared with nine positive investigations in 2004). Seven of the re-offending sites were part of complex clusters.

Nine accommodation sites (including two re-offending sites) were published on the EWGLI website during 2005 for failure to return Form $A$ or Form $B$ reports on time, or for failure to implement appropriate control measures within the required period (two further re-offending sites from 2005 were published in 2006). These sites were located in France (3 sites), Italy (2), Turkey (2), Czech Republic (1), and Greece (1). This represents a significant reduction from the 27 site names published during 2003, but is up from only four in 2004.

Under the EWGLI guidelines, there is no requirement to investigate sites associated with a single case report. Some countries do however conduct investigations, and EWGLINET is always interested in collating these results. During 2005, investigation reports were received for 114 such sites. Seventy $(61 \%)$ were reported positive for Legionella (at concentrations equal to or greater than 1,000 cfu/litre [1]), while 44 sites (39\%) were reported negative.

\section{Discussion}

2005 produced EWGLINET's highest ever case load, continuing the trend of increasing case numbers observed in previous years, and thought to be due in part to strengthening surveillance systems in collaborating countries. This increase in cases has coincided with a decreased case fatality rate (dropping to $3.8 \%$ in 2005), possibly due to a greater number of less seriously ill cases being diagnosed and notified to EWGLINET.

The number of cases per cluster has continued to decrease, indicating the ongoing success of the European guidelines [1]. The guidelines become operational at an accommodation site after it is associated with a first case, when the site is issued with a good practice checklist. With the cooperation of hoteliers, this could act in many instances to prevent subsequent cases. If a second case does occur, the European guidelines require the site to be thoroughly investigated. EWGLINET would expect only an infrequent number of subsequent cases to arise at a site that had been properly investigated (this can occur by chance as not every site associated with a case is the source of the infection), and this is borne out by the decreasing number of cases per cluster.

However, at the same time as the cluster size is decreasing, EWGLINET is experiencing an increase in the number of re-offending sites. The sites for 2005 were spread out across seven countries, and EWGLINET would expect some re-offences to occur each year by chance (when cases stay at accommodation sites unrelated to their illness, but which have been previously investigated under the guidelines). However, the number of re-offenders in Turkey is unusually high at 11 , especially in comparison with the four sites that re-offended in 2004, and extra care should be taken to ensure the guidelines are being properly applied.

The proportion of Turkey's cases associated with clusters has risen in 2005 to over $50 \%$. This is disappointing for the scheme, as the 2004 percentage (44\%) was a vast improvement from the high of $71 \%$ (in 2002). EWGLINET wishes Turkey to continue encouraging hoteliers to achieve high standards of water maintenance and Legionella control programmes in their buildings.

Overall, the results from EWGLINET are very positive. Of the clusters that were detected, $36.6 \%$ would not have been recognised without the scheme. EWGLI is facing some uncertainty throughout 2006 and 2007, and it is possible that the scheme may move to the European Centre for Disease Prevention and Control (ECDC). Whatever the future brings, every effort will be made to ensure that this successful scheme continues to grow and improve, and to provide a valuable public health service.

\section{Acknowledgements}

This work is funded by the European Commission Health and Consumer Protection Directorate-General and supported by the European Centre for Disease Prevention and Control (ECDC).We would like to thank all the collaborators* for reporting their cases and all the people involved in public health control and prevention programs for travel-associated legionnaires' disease.

* The list of EWGLI collaborators is available at the following URL address: http://www.ewgli.org/collaborators.htm

Errata: The site in Hungary classified as a re-offending site had not in fact been previously investigated under the European guidelines. The authors apologise for misclassifying this site in the initial online version of the paper. The correction was made in the article on 22 February 2007.

In Table 2, the part of Cyprus which the cluster was associated with was mistakenly not labelled as an EU country. A correction was made on September 32007.

\section{References}

1. European Working Group for Legionella Infections. European Guidelines for Control and Prevention of Travel Associated Legionnaires' disease. 2002: PHLS London and http://www.ewgli.org

2. Ricketts KD, McNaught B, Joseph CA. Travel-associated Legionnaires' disease in Europe: 2004. Euro Surveill. 2006;11(4):107-10. Available from: http://www. eurosurveillance.org/em/v11n04/1104-227.asp

Citation: Ricketts K, McNaught B, Joseph C, European Working Group for Legionella Infections, . Travel-associated legionnaires' disease in Europe: 2005. Euro Surveill 2007;12(1)[Epub ahead of print]. Available online: http://www.eurosurveillance. org/em/v12n01/1201-228.asp 